PAPER

Shock oscillation in highly underexpanded jets

To cite this article: Xiao-Peng Li et al 2018 Chinese Phys. B 27094705

View the article online for updates and enhancements. 


\title{
Shock oscillation in highly underexpanded jets*
}

\author{
Xiao-Peng Li(李晓鹏) $)^{1, \dagger}$, Rui Zhou(周芯) $)^{2}$, Xiao-Ping Chen(陈小平) $)^{3}$, \\ Xue-Jun Fan(范学军) ${ }^{4}$, and Guo-Shan Xie(谢国山) ${ }^{1}$ \\ ${ }^{1}$ China Special Equipment Inspection and Research Institute, Beijing 100029, China \\ ${ }^{2}$ Laboratory of Computational Physics, Institute of Applied Physics and Computational Mathematics, Beijing 100094, China \\ ${ }^{3}$ Key Laboratory of Fluid Transmission Technology of Zhejiang Province, Zhejiang Sci-Tech University, Hangzhou 310018, China \\ ${ }^{4}$ State Key Laboratory of High Temperature Gas Dynamics, Institute of Mechanics, Chinese Academy of Sciences, Beijing 100190, China
}

(Received 26 February 2018; revised manuscript received 6 June 2018; published online 28 August 2018)

The oscillatory motions of shocks in highly underexpanded jets with nozzle pressure ratios of 5.60, 7.47, 9.34, and 11.21 are quantitatively studied by using large eddy simulation. Two types of shock oscillations are observed: one is the Mach disk oscillation in the streamwise direction and the other is the shock oscillation in the radial direction. It is found that the Mach disk moves quickly in the middle of the oscillatory region but slowly at the top or bottom boundaries. The oscillation cycles of Mach disk are the same for different cases, and are all dominated by an axisymmetric mode of 5.298 $\mathrm{kHz}$. For the oscillation in the radial direction, the shocks oscillate more toward the jet centerline but less in the jet shear layer, and the oscillation magnitude is an increasing function of screech amplitude. The cycles of the radial shock oscillation switch randomly between the two screech frequencies for the first two cases. However, the oscillation periodicity is more complex for the jets with high nozzle pressure ratios of 9.34 and 11.21 than for the jets with the low nozzle pressure ratios of 5.6 and 7.47. In addition, the shock oscillation characteristics are also captured by coarse mesh and Smagorinsky model, but the coarse mesh tends to predict a slower and weaker shock oscillation.

Keywords: highly underexpanded jets, shock oscillation, large eddy simulation

PACS: 47.27.wg, 47.60.Kz, 47.40.Nm, 47.27.ep

DOI: $10.1088 / 1674-1056 / 27 / 9 / 094705$

\section{Introduction}

Subsonic jets ${ }^{[1-5]}$ and supersonic underexpanded jets $^{[6-10]}$ have long been the subject of numerous experimental, theoretical, and numerical studies because of their widespread applications in energy and propulsion systems. In particular, the flow field of an underexpanded jet is mainly characterized by the quasi-periodic shock waves. These shocks interact with turbulent structures along the jet shear layer, producing screech ${ }^{[11,12]}$ and broadband shockassociated noise ${ }^{[13,14]}$ in addition to the classical mixing noise. ${ }^{[6]}$ In particular, the screech is a very intense tonal noise component that may cause structural damage and fatigue failure of high-speed vehicle components, and is believed to be associated with the oscillation of the shock system formed inside the jet. ${ }^{[15-17]}$ Therefore, revealing the unsteady shock behavior is vital to accurately understand the flow physics of underexpanded jets, which will benefit greatly the jet mixing enhancement design and noise reduction.

Prandtl ${ }^{[9]}$ was the first to determine the shock cell length of a supersonic underexpanded jet based on the linear inviscid theory. Pack ${ }^{[10]}$ corrected Prandtl's theory (1904) by introducing the velocity on the bounding stream-lines, and found that theoretical and experimental values for the shock cell length of circular jets are in good agreement. Powell ${ }^{[11]}$ observed experimentally that supersonic underexpanded jets will produce a screech tone, and proposed a formula to calculate the screech frequency based on the shock spacing. After these previous work, plenty of research efforts ${ }^{[12-24]}$ have been devoted to studying the underexpanded jets, and the knowledge of the steady shock structures and acoustic properties have been well gained. A comprehensive summary of the supersonic jet noise can be found in the review papers by Tam ${ }^{[6]}$ and Raman. ${ }^{[7]}$ However, the information about the unsteady shock motions, i.e., the oscillation characteristics of shock cells in an underexpanded jet when the jet is fully developed, is still limited. This is probably because the shocks generally oscillate at high frequency $(\sim 10 \mathrm{kHz})$ and within a narrow spatial range, which makes it difficult to perform the quantitative measurements. To the authors' best knowledge, there exist only Refs. [12], [15], [20], and [21] that involve the quantitative measuring of the shock oscillations of underexpanded jets. In particular, Sherman et al. ${ }^{[12]}$ investigated the shock distortion duing screech by using high-speed schlieren, and found the coincidence between the oscillation and screech frequencies. Panda ${ }^{[15]}$ developped an optical shock detection technique based on laser light scattering, and reported the shock motion characteristic in underexpanded jets. An analytical model was also proposed by Panda, ${ }^{[15]}$ and the predicted oscillation amplitude of the first shock agrees reasonably with the

* Project supported by the National Natural Science Foundation of China (Grant No. 11602028) and the Science and Technology Project of General Administration of Quality Supervision Inspection and Quarantine of China (Grant Nos. 2017QK119 and 2017QK188).

†Corresponding author. E-mail: 1xpyfy@163.com

(C) 2018 Chinese Physical Society and IOP Publishing Ltd

http://iopscience.iop.org/cpb http://cpb.iphy.ac.cn 
measured data. André et al. ${ }^{[20,21]}$ developed a shock-tracing procedure by making use of schlieren images and a patternmatching algorithm to study the unsteady shock behavior in both stable and unstable underexpanded jets.

High-resolution modeling, e.g., large eddy simulation (LES) or direct numerical simulation (DNS), can provide more information about the flow characteristics of an underexpanded jet. Singh and Chatterjee ${ }^{[25]}$ analyzed the peak frequencies of an underexpanded jet with a fully expanded jet Mach number of 1.19 using compressible LES. Berland et $a l .{ }^{[26]}$ studied the generation mechanism of sceech tones in a planar supersonic jet by using the LES. Vuorinen et al. ${ }^{[27]}$ performed the LES modeling of underexpanded jets at different nozzle pressure ratios (NPRs), and further compared the flow differences between the methane and nitrogen jets. ${ }^{[28]}$ Hamzehloo and Aleiferis ${ }^{[29]}$ focused on the flow characteristics of hydrogen underexpanded jets, and then compared the mixing characteristics of hydrogen jets with methane jets at various NPRs. ${ }^{[30]}$ Li et al. ${ }^{[31]}$ investigated the differences in flow structure and screech characteristic between the hydrogen and nitrogen underexpanded jets by using the LES. A better understanding of the underexpanded jets, including the screech generation, ${ }^{[25,26]}$ the initial shock dynamics and jet development, ${ }^{[27-29]}$ the quasi-steady shock and jet structures, ${ }^{[27-31]}$ and the mixing characteristics of different fuel jets, ${ }^{[28,30,31]}$ is thus gained. However, the physical picture of the unsteady shock motion is still not clear, which is little referred in those studies. In addition, the nozzle pressure ratio $\left(\mathrm{NPR}, P_{0} / P_{\infty}\right)$, which is the ratio of the nozzle total pressure $\left(P_{0}\right)$ to the ambient static pressure $\left(P_{\infty}\right)$, dominates the near-field shock structures as well as the screech in supersonic underexpanded jets. The NPRs invesigated in Refs. [12], [15], [20], and [21] are relatively small. For example, the jets examined by Panda ${ }^{[15]}$ take NPR values of 2.4 and 3.3 , while the NPRs in André et al. ${ }^{[20]}$ are 2.27 and 2.54. Those jets are generally classified as moderately underexpanded, and exhibit oblique shock patterns in the near-field region. However, a normal shock, i.e., Mach disk, forms when NPR increases beyond 3.85, and the jet is then claasified as a highly underexpanded jet. ${ }^{[8,32-34]}$ Considering the great difference in wave structure between moderately and highly underexpanded jets, an in-depth insight into the shock structure and its unsteady motion characteristic in highly underexpanded jets is necessary.

The current study expands an earlier work ${ }^{[35]}$ by the authors, where time evolution and instability of highly underexpanded jets were investigated by the LES. The focus of this study is to reveal the unsteady shock behavior in highly underexpanded jets and understand its physics based on highresolution LES data. The grid independence is investigated, and the time-avergaed shock structrues are compared with the available literature data to verify the present LES modeling. Instantaneous contours of density gradient are used to visualize the shock motion qualitatively. Then, instantaneous profiles of pressure along the jet centerline are examined to quantitatively assess the motion of Mach disk in the streamwise direction. The shock motion in the radial direction is addressed based on the jet boundary tracking accorrding to the radial proflies of vorticity or subgrid turbulent kinetic energy (TKE). Pressure measurement and spectrum analysis are also performed to relate the screech properties to shock oscillations. In addition, the effect of grid and the subgrid scale (SGS) turbulence models employed in the LES to reveal the shock oscillation characteristics is investigated. Quantitative findings of the shock motion are thus achieved, and this study may provide a new viewpoint to understand the shock behavior of underexpanded jets.

\section{Computational description}

This study is an extended analysis of the LES results provided in Ref. [35], where the computational method has been presented in detail. However, a brief description of the simulation setup is also included here for completeness.

\subsection{Numerical methods}

The governing equations used in the present LES are three-dimensional (3D) filtered compressible Navier-Stokes equations, including the following conservation equations for mass, momentum, energy, and species:

$$
\begin{aligned}
& \frac{\partial \bar{\rho}}{\partial t}+\frac{\partial \bar{\rho} \tilde{u}_{i}}{\partial x_{i}}=0 \\
& \frac{\partial \bar{\rho} \tilde{u}_{i}}{\partial t}+\frac{\partial \bar{\rho} \tilde{u}_{i} \tilde{u}_{j}}{\partial x_{j}}=-\frac{\partial \bar{p}}{\partial x_{i}}+\frac{\partial \tilde{\tau}_{i j}}{\partial x_{j}}-\frac{\partial \tau_{i j}^{\mathrm{sgs}}}{\partial x_{j}}+\frac{\partial D_{i j}^{\mathrm{sgs}}}{\partial x_{j}} \\
& \frac{\partial \tilde{\rho} \tilde{h}_{\mathrm{s}}}{\partial t}+\frac{\partial \tilde{\rho} \tilde{u}_{j} \tilde{h}_{\mathrm{s}}}{\partial x_{j}}=\frac{\partial \bar{p}}{\partial t}+\frac{\partial \tilde{u}_{j} \tilde{\tau}_{i j}}{\partial x_{i}}-\frac{\partial \bar{q}_{i}}{\partial x_{i}}-\frac{\partial H_{i}^{\mathrm{sgs}}}{\partial x_{i}}+\frac{\partial \sigma_{i}^{\mathrm{sgs}}}{\partial x_{i}} \\
& \frac{\partial \bar{\rho} \tilde{Y}_{k}}{\partial t}+\frac{\partial \bar{\rho} \tilde{u}_{j} \tilde{Y}_{k}}{\partial x_{j}}=\frac{\partial}{\partial x_{j}}\left(\bar{\rho} \tilde{D_{k m}} \frac{\partial \tilde{Y}_{k}}{\partial x_{j}}\right)-\frac{\partial \Phi_{k, j}^{\mathrm{sgs}}}{\partial x_{j}}-\frac{\partial \theta_{k, j}^{\mathrm{sgs}}}{\partial x_{j}}
\end{aligned}
$$

where $\rho$ is the density, $u_{i}$ is the velocity in the $x_{i}$ direction, $p$ is the pressure, $\tau_{i j}$ is the viscous stress tensor, $h_{\mathrm{s}}$ is the sensible enthalpy, $q_{i}$ is the heat flux vector, $Y_{k}$ is the mass fraction, and $D_{k m}$ is the equivalent binary mass diffusivity. The pressure is computed from the equation of state

$$
\bar{p}=\bar{\rho} R \tilde{T}+R_{\mathrm{u}} \sum T^{\mathrm{sgs}},
$$

where $\tilde{T}$ is the temperature, $R$ is the gas constant of the mixture gas, and $R_{\mathrm{u}}$ is the universal gas constant. The terms with superscript sgs in Eqs. (1)-(5) denote the sub-grid quantities, and are modeled with the sub-grid scale (SGS) turbulent kinetic energy one-equation model. ${ }^{[36]}$ The thermodynamic and transport properties of individual species, such as the enthalpy per 
unit mass $h_{k}$ and the specific heat at constant pressure $c_{p k}$, are calculated based on NIST-JANAF thermo-physical and transport database. ${ }^{[37]}$ The dynamic viscosity $\mu_{k}$ is computed by Sutherland's law. The Schmidt number $S c$ and the turbulent Prandtl number $P r_{t}$ in the species concentration and energy equations are both assumed to be a constant of 1.0.

The density-based compressible solver astroFoam, ${ }^{[31,35]}$ which is developed based on the basic rhoCentralFoam solver ${ }^{[38]}$ distributed with OpenFOAM v2.3.0, is used to solve the above equations. Comparing with the original rhoCentralFoam solver, two aspects are modified to create the astroFoam solver. First, multiple species transport and multicomponent diffusion (Eq. (4)) are added into the astroFoam solver to model more realistic mixing and reacting flows. Second, the energy equation (Eq. (3)) is solved for senible enthalpy in astroFoam instead of total energy in rhoCentralFoam to include the chemical reaction and species transport terms more easily. In addition, the convection-diffusion equation is solved by semi-discrete KT scheme ${ }^{[39]}$ for capturing shock and solving turbulence in astroFoam. A normalized variable diagram (NVD) scheme ${ }^{[40,41]}$ is used to reconstruct the primitive values at faces to obtain the second order accuracy. Time integration is carried out by the Crank-Nicholson scheme, ${ }^{[41]}$ which is second order accuracy in time.

\subsection{Computational domain and grid}

Figure 1(a) shows the computational domain used in the current LES, which mainly consists of a box of size $50 \mathrm{~mm} \times 100 \mathrm{~mm} \times 50 \mathrm{~mm}$ in $x, y$, and $z$ Cartesian coordinate directions. Previous studies ${ }^{[6,7,11,16]}$ indicated that the sound waves originating from the down stream will propagate upstream to change the initial shear layer structures at the nozzle exit, which will influence the development of the jet shear layer in the down stream further. However, the priori knowledge of nozzle exit conditions is usually difficult to obtain in many practical applications. In the present study, a convergent nozzle geometry is included in the upstream part of the computational domain. The nitrogen jet (with total pressure $P_{0}$ and total temperature $T_{0}$ ) is injected into the quiescent air (with static pressure $P_{\infty}$ and static temperature $T_{\infty}$ ) from the convergent nozzle of $20.0 \mathrm{~mm}$ in height. The entrance and exit diameters of the nozzle are $d=8.0 \mathrm{~mm}$ and $D=2.0 \mathrm{~mm}$, respectively.

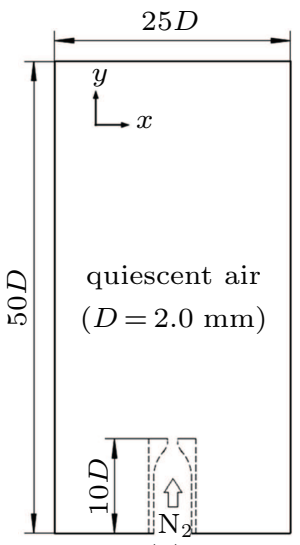

(a)

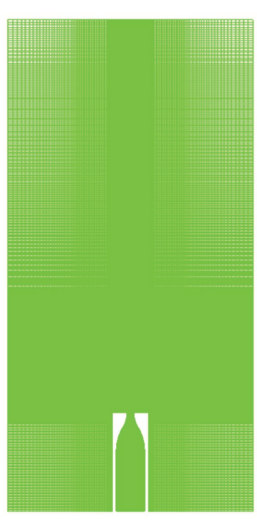

(b)

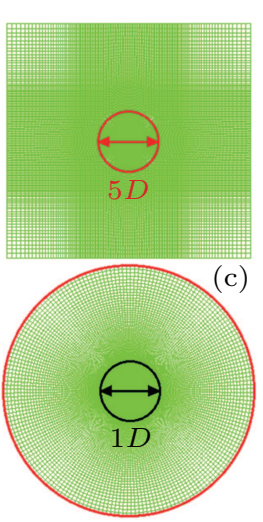

(d)
Fig. 1. (color online) Simulation setup showing (a) computational model and (b)-(d) computational grid: (b) side view at $z / D=0$, (c) top view at $y / D=0$, (d) close-up view at nozzle exit.

As indicated by the previous studies, ${ }^{[27-31,42-46]}$ the spatial resolution in LES of supersonic jets needs to be rather high. Figures 1(b)-1(d) show the hexahedral, block-structured grid used in the present LES. The grid contains a refinement region of high resolution, covering the jet core and the jet shear layers. In the refinement region, two levels of grid resolutions, i.e., fine and coarse, are used to characterize the grid dependence of the solution. The fine and coarse meshes contain about 27.3 and 13.0 million cells, respectively, and their resolutions are similar to those used in previous LES of supersonic jets, ${ }^{[27,43-46]}$ which are summarized in Table 1 . On the other hand, coarse cell sizes with a resolution of $1.0 \mathrm{~mm}$ in the far field and $0.5 \mathrm{~mm}$ at the outflow boundaries are used to avoid wave reflections by introducing additional dissipation.

Table 1. Grid resolution comparison in the near field of supersonic jets.

\begin{tabular}{|c|c|c|c|c|c|c|c|}
\hline Grid & $\mathrm{D} / \mathrm{mm}$ & $\Delta r_{\min }$ & $\Delta r_{\max }$ & $\Delta y_{\min }$ & $\Delta y_{\max }$ & $\mathrm{Re}$ & Total $/ 10^{6}$ \\
\hline Fine & 2.0 & $D / 200$ & $D / 52$ & $D / 67$ & $D / 25$ & $\sim 10^{5}$ & 27.3 \\
\hline Coarse & 2.0 & $D / 200$ & $D / 25$ & $D / 50$ & $D / 20$ & $\sim 10^{5}$ & 13.0 \\
\hline Vuorinen et al. ${ }^{[27]}$ & 1.4 & $D / 70$ & $D / 50$ & $D / 35$ & $D / 25$ & $\sim 10^{5}$ & 12.0 \\
\hline Gorle et al. ${ }^{[43]}$ & 2.0 & $D / 100$ & $D / 50$ & $D / 100$ & $D / 25$ & $\sim 10^{5}$ & 17.4 \\
\hline Dauptain et al. ${ }^{[44]}$ & 25.4 & $D / 35$ & $D / 30$ & $D / 35$ & $D / 30$ & $\sim 10^{6}$ & 22.0 \\
\hline Rana et al. ${ }^{[46]}$ & 4.0 & $D / 33$ & $D / 33$ & $D / 33$ & $D / 33$ & $\sim 10^{4}$ & 9.2 \\
\hline
\end{tabular}

\subsection{Initialization and boundary conditions}

The quiescent air is the mixture of $76.699 \%$ nitrogen and $23.301 \%$ oxygen in mass fraction. Initially the temperature, pressure, density, and velocity of the ambient air are all uniform, i.e., $T_{\infty}=300 \mathrm{~K}, P_{\infty}=101325 \mathrm{~Pa}, \rho_{\infty}=1.17 \mathrm{~kg} / \mathrm{m}^{3}$, and $U_{\infty}=0$. Four different simulations with NPRs of 5.60, $7.47,9.34$, and 11.21 are carried out to examine the shock behavior in underexpanded jets. The flow conditions at the nozzle exit are close to sonic conditions for different jets, and are summarized in Table 2 in detail. The stagnation condition 
for temperature and pressure is employed at the high-pressure nozzle inlet, while the velocity is treated with a zero-gradient condition. All walls are treated as no-slip, adiabatic condition. At the top of the computational domain and on the four free surfaces, an open boundary condition is used, i.e., all flow parameters are treated as the zero-gradient for outflow and fixed ambient values in the case of backflows.

The time step $\Delta t$ in the LES of supersonic flows is generally small. Kawai and Lele ${ }^{[45]}$ used a time step satisfying $\Delta t \cdot U_{\infty} / D=9.6 \times 10^{-4}$ in the LES of a sonic jet in supersonic cross flow. Liu et al. ${ }^{[42]}$ employed a maximum Courant-Friedrichs-Lewy (CFL) number of 0.4 while Dauptain et al. ${ }^{[44]}$ used 0.7 in their LES modeling of supersonic underexpanded jets. The time steps used by Hamzehloo and Aleiferis $^{[30]}$ in the LES of methane underexpanded jets are both between $2 \times 10^{-8} \mathrm{~s}$ and $5 \times 10^{-8} \mathrm{~s}$. The computational time step used in the present LES is similar to those used in previous LES studies, ${ }^{[30,42,44,45]}$ and takes a value approaching $\Delta t=1.37 \times 10^{-8} \mathrm{~s}$, which gives a $\Delta t \cdot a / D$ of $2.42 \times 10^{-3}$ and is limited by the maximum CFL number of 0.6. The flowthrough time (FTT) for jets washing out the computational domain in the streamwise direction is around $0.5 \mathrm{~ms}=200 t_{0}$, with $t_{0}=2.5 \times 10^{-6} \mathrm{~s}=2.5 \mu \mathrm{s}$. The total simulation duration is thus set to be $4 \mathrm{FTT}=2.0 \mathrm{~ms}=800 t_{0}$. The instantaneous results are saved every $2 t_{0}$, and turbulent statistics are collected for the last three flow-through times (3FTT, 200t $t_{0}$ to $800 t_{0}$ ).

Table 2. Flow parameters and simulation conditions.

\begin{tabular}{lcccccc}
\hline Property & Symbol & Case 1 & Case 2 & Case 3 & Case 4 & Units \\
\hline Mach number at nozzle exit & $M_{1}$ & 1.0 & 1.0 & 1.0 & 1.0 & - \\
Static pressure at nozzle exit & $P_{1}$ & 0.3 & 0.4 & 0.5 & 0.6 & $\mathrm{MPa}$ \\
Stagnation pressure & $P_{0}$ & 0.57 & 0.76 & 0.95 & 1.14 & $\mathrm{MPa}$ \\
Static temperature at nozzle exit & $T_{1}$ & 300.0 & 300.0 & 300.0 & 300.0 & $\mathrm{~K}$ \\
Reynolds number at nozzle exit & $R e_{1}$ & 1.36 & 1.82 & 2.27 & 2.73 & $10^{5}$ \\
Static pressure ratio & $P_{1} / P_{\infty}$ & 2.96 & 3.95 & 4.93 & 5.92 & - \\
Nozzle pressure ratio (NPR) & $P_{0} / P_{\infty}$ & 5.60 & 7.47 & 9.34 & 11.21 & - \\
\hline
\end{tabular}

\section{Numerical validation}

\subsection{Mesh independence and convergence}

In order to establish the fidelity of the LES results, a grid convergence study has been conducted. The LES of underexpanded jet at NPR $=5.60$ is carried out by using the two different grids, i.e., the coarse and fine meshes. It is important
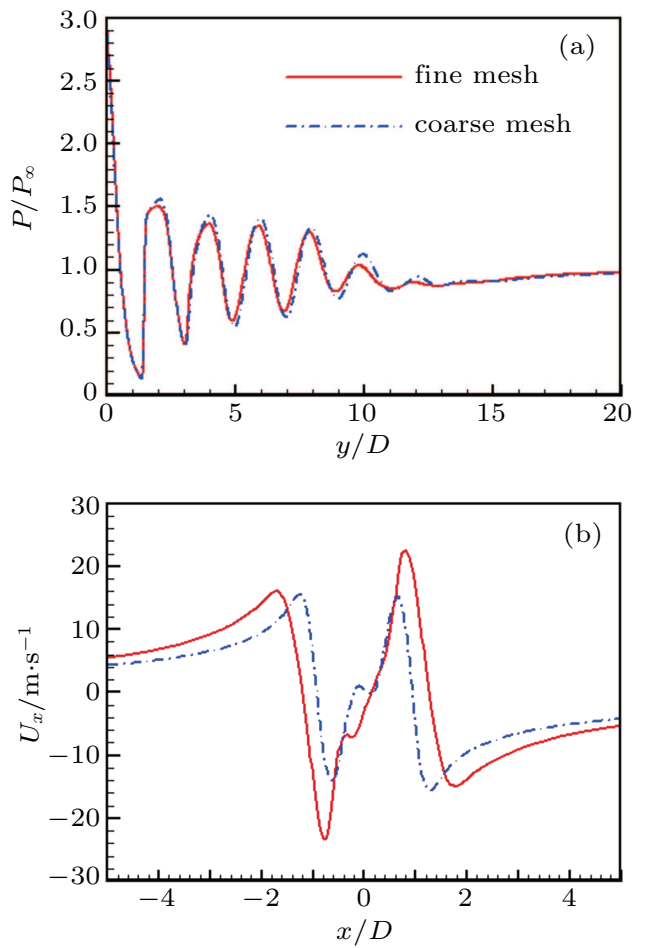

Fig. 2. (color online) Grid independence analysis for NPR $=5.60$, showing (a) axial pressure profiles and (b) radical profiles of mean normal velocity at $y / D=10$ to capture the quasi-periodic shock structure as its interaction with the jet shear layer is responsible for the shock screech phenomenon. Figure 2(a) shows the mean pressure profiles along the jet centerline. As can be seen, the agreement over the first four shock cells is very good for the two meshes. The turbulence properties of the jets also need taking great concern. The radical profiles of the mean normal velocity, $U_{x}$, are compared for the two meshes in Fig. 2(b). The overall trends are similar for the two profiles, but the coarse mesh seems to, as expected, restrain the development of turbulence because of the larger grid dissipation, which is indicated by a relatively narrow jet boundary. Therefore, the fine mesh is mainly used for the results discussed subsequently, while the results obtained by the coarse mesh are only presented in Subsection 4.4.

\subsection{Time-averaged shock structures}

Figures 3(a) and 3(c) show the time-averaged density gradients for NPR $=5.60$ and 9.34 obtained by LES, respectively. As can be seen, the typical wave structures in the near field of a highly underexpanded jet, including the Mach disk, intercepting shock, triple point, reflected shock, and slip lines that have been confirmed by previous experimental and numerical studies, ${ }^{[8,17,22-24,27-34]}$ are all well captured by the LES. The predicted shock structures are also in good agreement with those in the schlieren photographs shown in Figs. 3(b) and 3(d) under the same NPR. In particular, the locations of the Mach disk, the first and the second reflected shocks on the jet boundary (marked by lines) predicted by the LES are reasonably comparable to the measurements. 


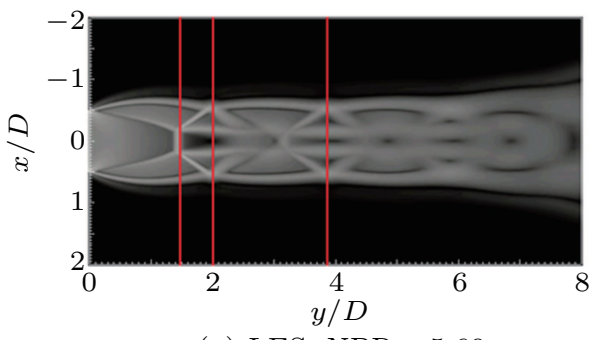

(a) LES, NPR $=5.60$

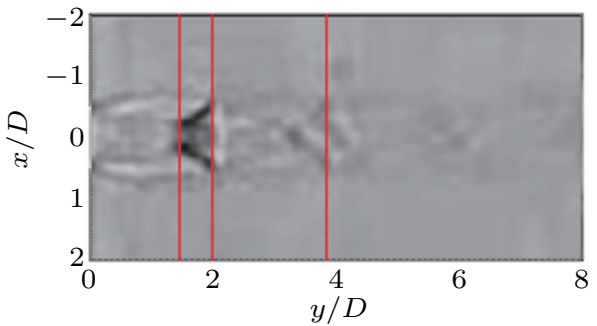

(b) $\operatorname{Exp}, \mathrm{NPR}=5.60$

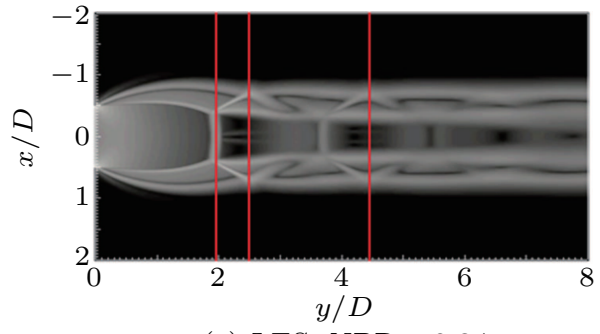

(c) LES, NPR $=9.34$

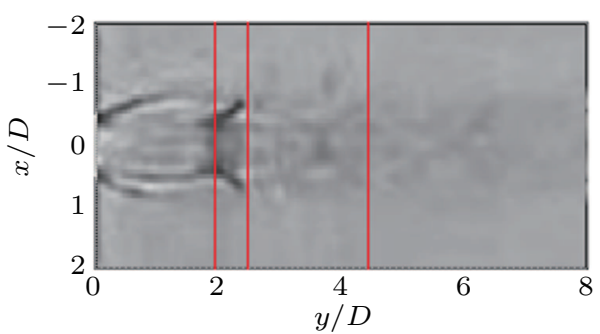

(d) Exp, NPR $=9.34$

Fig. 3. (color online) Comparisons of time-averaged near-field shock structure between LES and experimental data for NPR of (a), (b) 5.60 and (c), (d) $9.34 .{ }^{[35]}$

Figure 4 shows the iso-surfaces of density gradient for $\mathrm{NPR}=5.60$, which presents the 3D formation and development of the near-field shocks. The jet boundary, the intercepting shock, and the reflected shock are circular in cross-section. The slip line surface (or shear layer) initially expands the down stream of the Mach disk, then rapidly contracts as observed experimentally by Mitchell et al. ${ }^{[23]}$ The flow is supersonic $(M a \sim 3.0)$ and possesses a low temperature $(T \sim 100 \mathrm{~K})$ and high velocity $\left(U_{y} \sim 700 \mathrm{~m} / \mathrm{s} \approx 2 U_{\mathrm{e}}, U_{\mathrm{e}}\right.$ is the nozzle exit velocity) in the vicinity of the Mach disk because of rapid expansion, and becomes subsonic $(M a \sim 0.2)$ immediately after the Mach disk. Then the flow is accelerated to sonic conditions through the circular duct bounded by the shear layer.
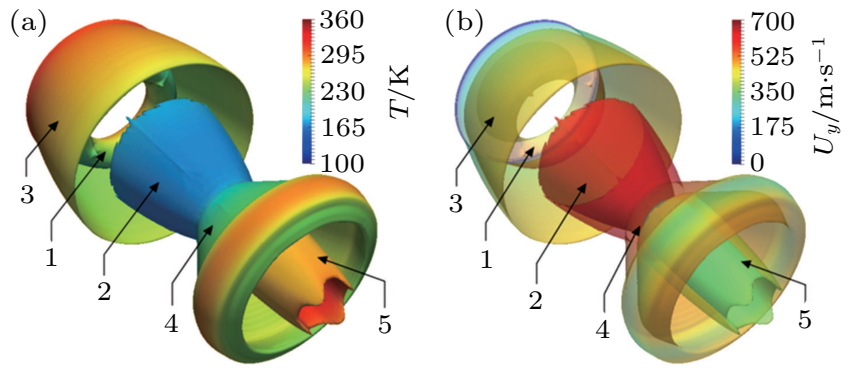

1- portion of expansion fan 3- jet boundary 5- shear layer 2 - intercepting shock 4 - reflected shock

Fig. 4. (color online) Iso-surfaces of density gradient of 3D near-field shock structures for NPR = 5.60: (a) solid view, (b) perspective view.

Figure 5(a) shows quantitative comparisons of timeaveraged density along the jet centerline between the LES results for NPR $=5.60$ and the measured data by Panda and Seasholtz ${ }^{[17]}$ at a similar NPR of 5.74. Note that $\rho_{\mathrm{j}}$ is the fully expanded jet density. Good agreement with the experimental data is observed for the first two shocks. However, roughly from the third shock, the LES results start to deviate from the measurements. This deviation may be attributed to the difference between $\rho_{\mathrm{j}} / \rho_{\infty}$ values used in the LES and the experimental values. On the other hand, the radial profiles of density near the Mach disk obtained by the LES are compared with the measurements in Fig. 5(b). The LES results agree well with the measured data at the first two streamwise positions, i.e., $y / D=0.9$ and 1.2. At $y / D=1.5$, the predicted density peaks at around $x / D=0.2$, which is smaller than the measured value of $x / D=0.25$. This observation suggests that the present LES presents a narrower Mach disk than the experiment. Similarly, the LES studies performed by Vuorinen et al. ${ }^{[27]}$ and Hamzehloo and Aleiferis ${ }^{[47]}$ reproduced the width of the Mach disk smaller than the measurements. The contributing factors, as indicated by Franquet et al. ${ }^{[8]}$ and explained by Hamzehloo and Aleiferis, ${ }^{[47]}$ can be the differences in nozzle geometry design and simulation setup (e.g., the turbulence levels at the nozzle exit) between the LES modeling and the experiment.

In addition, several previous numerical studies ${ }^{[48,49]}$ have indicated the existence of a recirculation zone formed immediately downstream of the Mach disk in an underexpanded jet. However, the recent quantitative velocity measurements for a highly underexpanded jet at an NPR of 4.2 by Mitchell et $a l .{ }^{[23]}$ showed that there is no experimental evidence for such a phenomenon. Figures 6(a) and 6(b) show the time-averaged profiles of pressure and streamwise velocities obtained by the present LES. As can be seen, the velocities for different jets indeed obtain the minimum values after the Mach disk, but there is no upstream velocity component (negative value) in the mean. This finding agrees reasonably with the prior experimental data, ${ }^{[22,23]}$ and also indicates that the present LES produces no recirculation region behind the Mach disk, which 
is in accordance with the recent LES study by Hamzehloo and Aleiferis. ${ }^{[30]}$
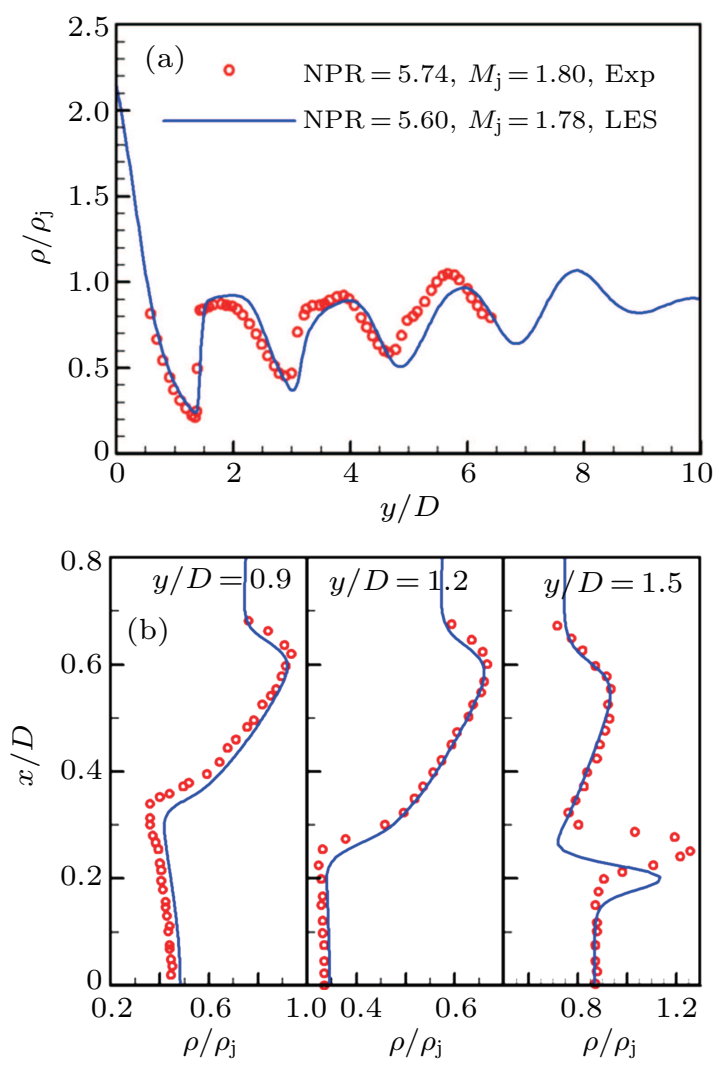

Fig. 5. (color online) Time-averaged density comparisons between LES and the measurements by Panda and Seasholtz: ${ }^{[17]}$ (a) axial profile, (b) radial profiles.
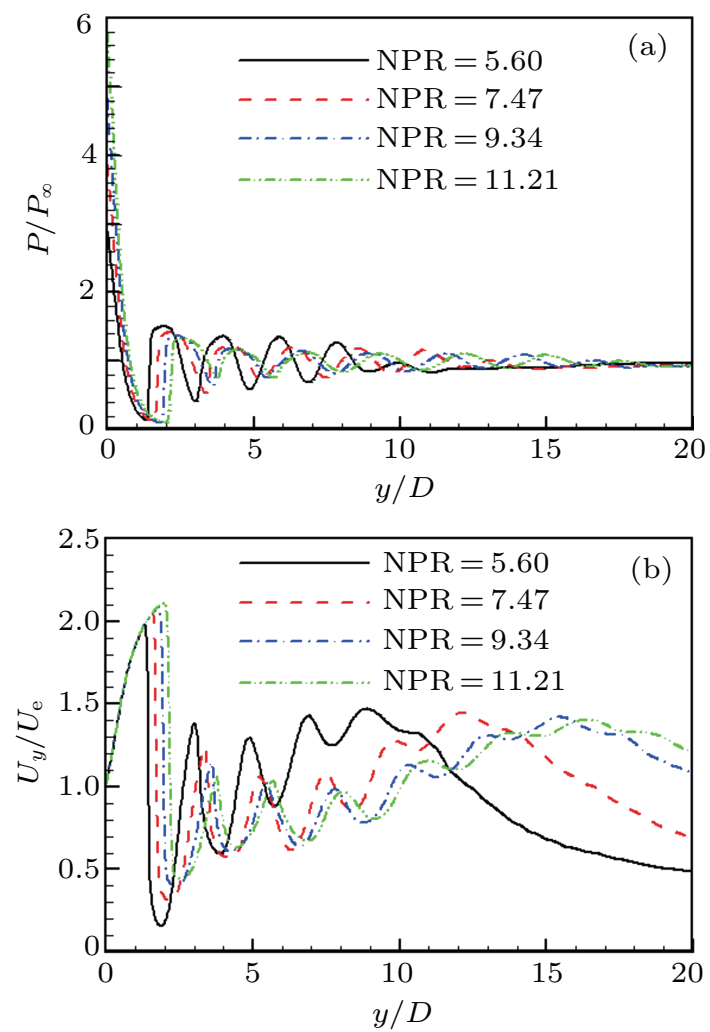

Fig. 6. (color online) Time-averaged flow properties along jet centerline: (a) non-dimensional pressure, (b) streamwise velocity.

\section{Results and discussion}

\subsection{Visualization of shock motion}

Figure 7 shows the time-averaged as well as instantaneous contours of density gradient on the midline plane at an NPR of 5.60 obtained by the LES. As also visualized through using schlieren photographs by Panda, ${ }^{[15]}$ the instantaneous flow fields are significantly different from the time-averaged ones, and undergo considerable distortion and show some oscillations. The distortion and oscillation progressively increase for the shocks formed further in the down stream from the nozzle exit. Especially at $5.0<y / D<7.5$, the intense oscillation occurs, where the jet boundary presents a left and right wavy motion associated with the deformation of the rhombus shape of the third shock cell. These observations are twodimensional (2D) impressions of a 3D helical motion of the jet where the jet core precesses about the jet centerline. After around $y / D=7.5$, the slip lines merge gradually with the jet boundary, making the fourth shock cell difficult to identify. Meanwhile, the organized large scale turbulent vortices are generated periodically and convected downstream with the flow. Panda ${ }^{[15]}$ observed that a new shock will occur when the turbulent disturbance convects over an existing shock wave in an axisymmetric jet with an NPR of 2.4, and regarded this phenomenon as shock splitting. For the NPR examined in this study, the shocks inside the jets are generally at a quasi-steady state, only oscillating in a limited spatial region without splitting.

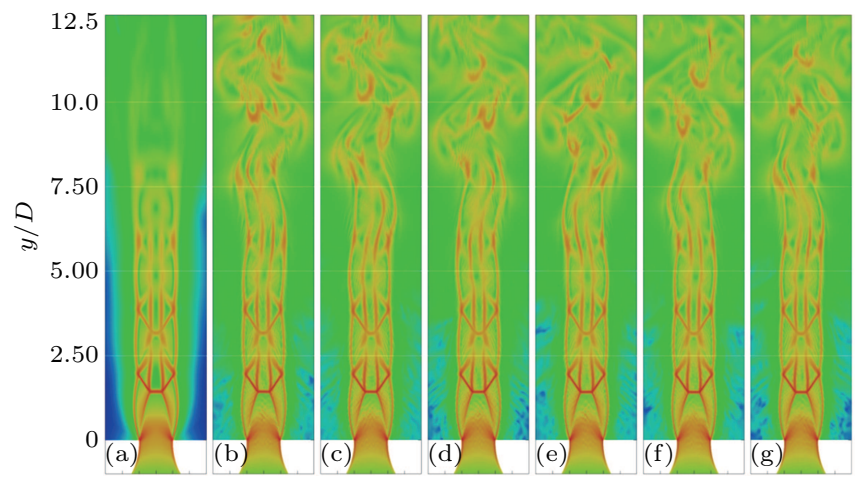

Fig. 7. (color online) (a) Time-averaged and (b)-(g) instantaneous density gradient at NPR of 5.60 corresponding to $t / t_{0}$ of (b) 468 , (c) 470 , (d) 472, (e) 474, (f) 476, and (g) 478, with one oscillation cycle.

Besides the noticeable wavy motion shown in Fig. 7, a careful examination of numerical schlieren photographs (i.e. density gradient) from frame to frame shows a vertical up and down motion for the shocks near the nozzle exit. In particular, figure 8 presents a close-up view of instantaneous flow structures in the near nozzle region at different time for an $\mathrm{NPR}=9.34$. The vertical oscillation of the first normal shock (i.e., Mach disk) can be identified. The second normal shock oscillates in the same manner as the Mach disk, being up and 
down alternately. Note that the initial formation and development of the near-nozzle shock structures and Mach disk in highly underexpanded jets have been examined in previous studies, see for example the measurements by Rogers et $a l .{ }^{[24]}$ and the recent LES modelings by Vuorinen et al. ${ }^{[27]}$ and Hamzehloo and Aleiferis. ${ }^{[29,30]}$ However, the vertical oscillation of Mach disk when the jet is fully developed, as shown in Fig. 8, is rarely reported. In addition, figures 7 and 8 each indicate that the shock oscillation amplitude is relatively small, implying that it is difficult to measure accurately by, for example, schlieren photographs. On the other hand, more details of the shock motion in underexpanded jets could be achieved based on the high-resolution LES data, especially the quantitative data to be addressed.

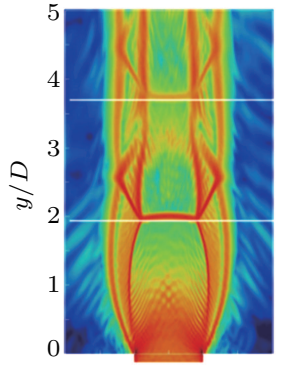

(a) $t=614 t_{0}$

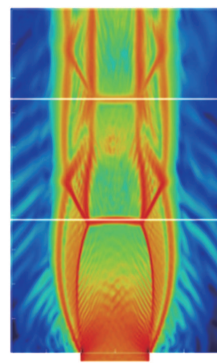

(b) $t=634 t_{0}$

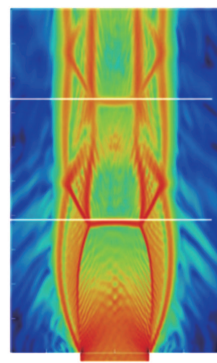

(c) $t=654 t_{0}$
Fig. 8. (color online) Instantaneous contours of density gradient for $\mathrm{NPR}=9.34$, showing the Mach disk is located at the highest, middle, and lowest position during one oscillation cycle in panels (a)-(c), respectively.

\subsection{Mach disk oscillation}

The key to quantitatively investigating the shock oscillation phenomenon is to accurately measure the position of the shocks and the distance over which they move. The shock is generally characterized by an intense jump in pressure as shown in Fig. 6(a), thus the instantaneous profiles of pressure along the jet centerline at different time are examined to quantify the Mach disk oscillation. Based on the detailed analysis of the pressure data, it is found that the Mach disk oscillates at the same cycle around $76 t_{0}=190 \mu$ s for different NPRs. Figure 9(a) shows the transference process of Mach disk from the lowest to the highest position during half an oscillation cycle for NPR $=5.60$. The time-averaged pressure profile is also included in Fig. 9(a), which denotes a much thicker Mach disk than the instantaneous one. In addition, the pressure curves are not uniformly distributed within the oscillation region, but appear to concentrate more near the lowest or highest positions. This indicates that the vertical oscillation of the Mach disk is nonlinear, i.e., moving quickly in the middle of the oscillation region but slowly at the top and bottom end. For other NPRs, the Mach disk oscillates similarly to that with an NPR of 5.60, i.e., undergoing the same oscillation cycle around $76 t_{0}=190 \mu$ s and moving more quickly in the middle region as shown in Fig. 9(b) for NPR $=9.34$.
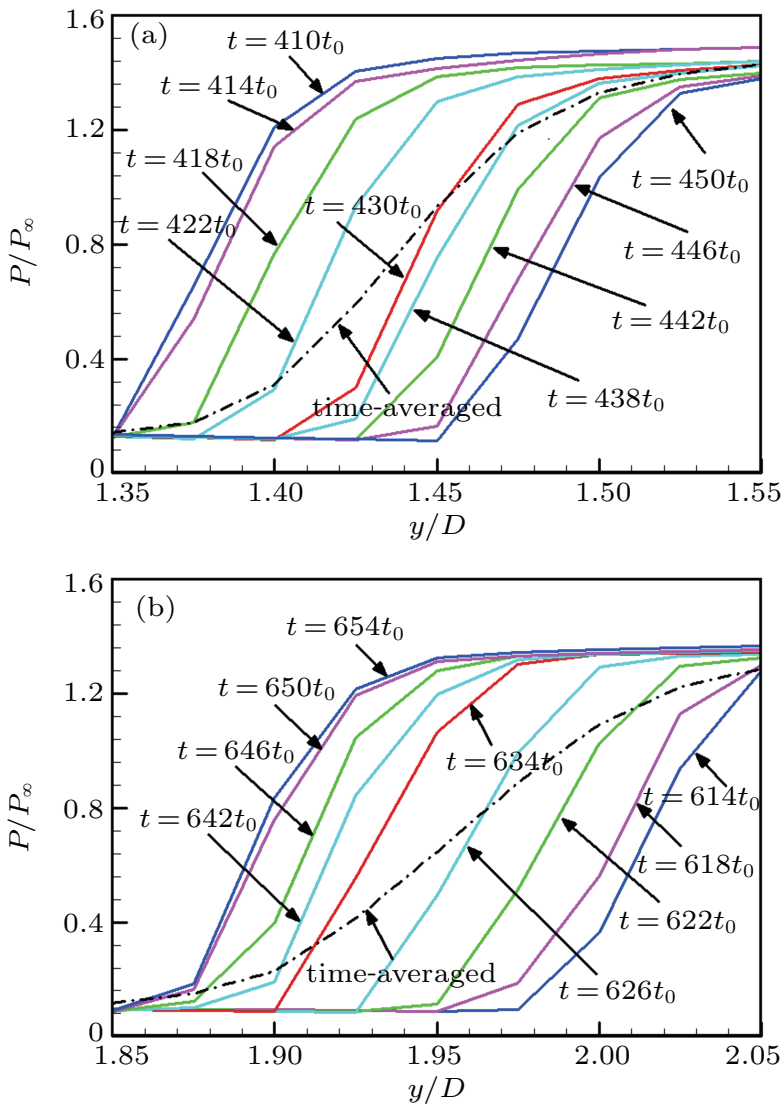

Fig. 9. (color online) Instantaneous pressures along the jet centerline, indicating the Mach disk motion in half oscillation cycle at NPR of (a) 5.60 and (b) 9.34
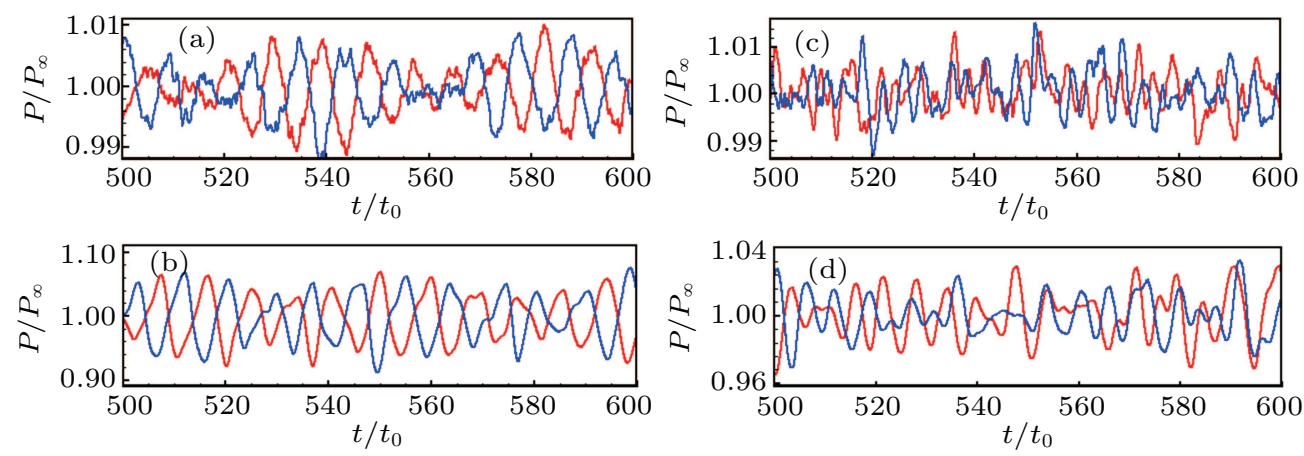

Fig. 10. (color online) Pressure history on either side of the jets at (a) NPR $=5.60, y / D=2$, (b) NPR $=5.60, y / D=6,(c)$ NPR $=9.34$ $y / D=2$, and (d) NPR $=9.34, y / D=6$. The red pressure curves are sampled at $x / D=1$ and $z / D=0$, while the blue pressure curves are sampled at $x / D=-1$ and $z / D=0$. 
The oscillation of Mach disk will bring in pressure fluctuating. Figure 10 shows the time histories of pressure near the jet shear layer at $y / D=2$ and 6 for NPR $=5.60$ and 9.34 , respectively. The pressure fluctuations on either side of jet appear to be correlated and opposite in phase at two different streamwise positions for NPR $=5.60$. At a higher NPR of 9.34, the relation between the two pressure signals is complex, opposite or consistent randomly in phase.

Figure 11 shows the spectra and relative phases in the near nozzle region of $y / D=2$ for different NPRs. As can be seen, there are several discrete peak frequencies in the pressure spectra. In particular, the frequency peaks circled with solid lines are the shock screech frequencies $f_{\mathrm{s}}$, and are equal to $37.086 \mathrm{kHz}, 34.437 \mathrm{kHz}, 31.787 \mathrm{kHz}$, and $29.801 \mathrm{kHz}$ for $\mathrm{NPR}=5.60,7.47,9.34$, and 11.21 , respectively. These screech frequencies decrease with NPR increasing, and have a relative phase angle close to $180^{\circ}$ (or $-180^{\circ}$ ), indicating
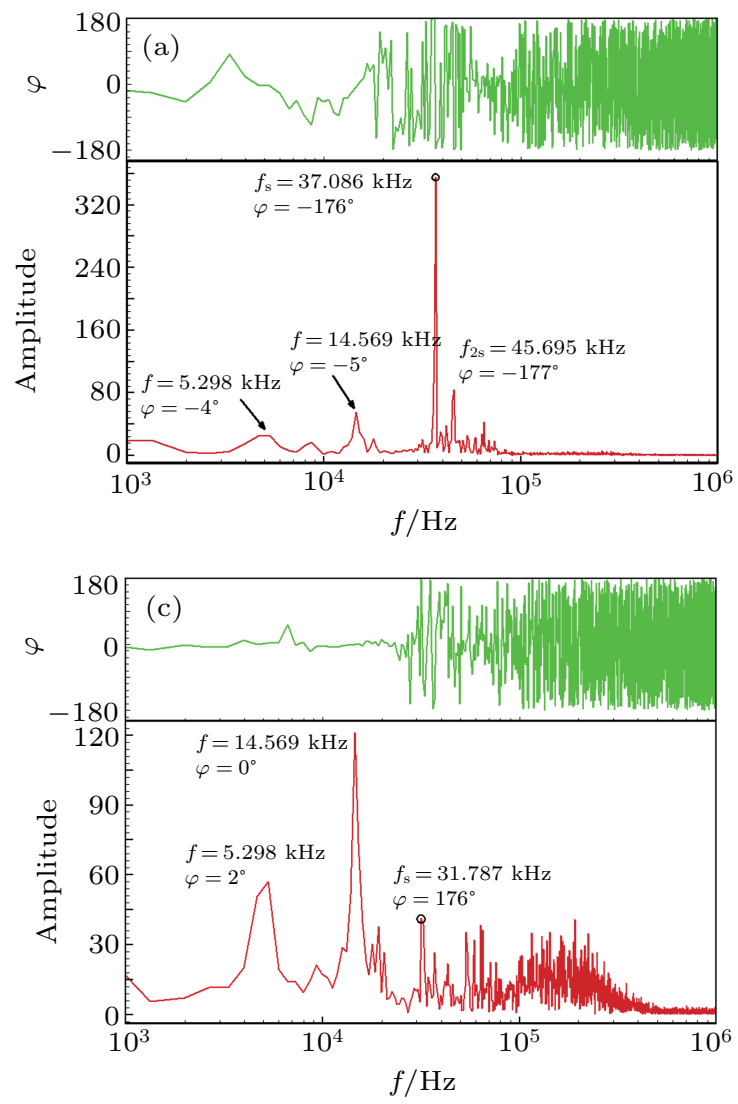

that the dominant modes of jets are all helical. ${ }^{[35]}$ Besides the peak frequencies of $37.086 \mathrm{kHz}$ and $34.437 \mathrm{kHz}$, other valid shock screech tones exist in the spectrum for NPR $=5.60$ and 7.47. The secondary shock screech tones take values of $f_{2 \mathrm{~s}}=45.695 \mathrm{kHz}$ and $40.397 \mathrm{kHz}$ for $\mathrm{NPR}=5.60$ and 7.47 , respectively, and are in helical mode as well with a relative phase angel close to $180^{\circ}$ (or $-180^{\circ}$ ). In addition, there are two axisymmetric modes with a relative phase angle close to $0^{\circ}$ in the spectrum, which takes the same frequency values of $f=5.298 \mathrm{kHz}$ and $14.569 \mathrm{kHz}$ for different NPRs. Remember that the Mach disk oscillation cycles for different NPRs are around $T_{\mathrm{soc}} \approx 76 t_{0}=190 \mu \mathrm{s}$, which is very close to the cycle of $T_{f}=1 / f=1 / 5.298 \times 10^{3} \approx 188.75 \mu$ s for the first axisymmetric mode of $5.298 \mathrm{kHz}$. Therefore, it can be inferred that the oscillations of Mach disk inside underexpanded jets at different NPRs are dominated by the same axisymmetric mode of $5.298 \mathrm{kHz}$.
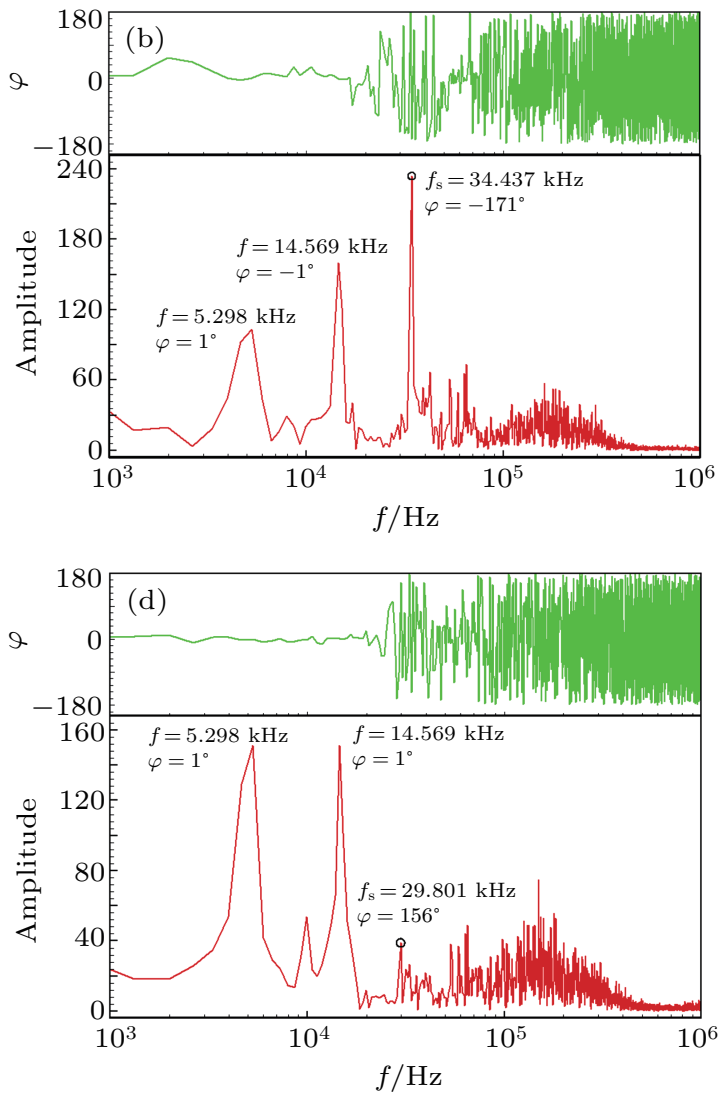

Fig. 11. (color online) Cross spectra and relative phases of pressure fluctuations on either side of jets at NPR of (a) 5.60, (b) 7.47, (c) 9.34, and (d) 11.21.

Note that the multiple screech tones have been observed experimentally in underexpanded rectangular ${ }^{[18]}$ and circular $^{[19]}$ jets. In particular, the fully expanded Mach number of the jets with multiple screech tones in Ref. [19] is around 1.8, which is close to those in the NPR $=5.60$ and 7.47 cases. ${ }^{[35]}$ In addition, Raman ${ }^{[7]}$ pointed out that the symmetric mode produced by a $2 \mathrm{D}$ convergent-divergent nozzle may be involved with the nozzle block design. The present LES results presented in the following Subsection 4.4 with different NPRs, grid resolutions, and subgrid scale turbulence models produce the same symmetric modes of $f=5.298 \mathrm{kHz}$ and $14.569 \mathrm{kHz}$. Therefore, the appearance of the axisymmetric mode of $f=5.298 \mathrm{kHz}$ associated with the Mach disk oscillation at the same frequency for different NPRs is probably related to the current nozzle geometry design. 


\subsection{Radial shock oscillation}

Figure 12(a) shows the time-averaged as well as instantaneous radical profiles of vorticity magnitude $\Omega$ for $\mathrm{NPR}=5.60$. In particular, the instantaneous curves are consistent with those in Figs. 7(b)-7(g), respectively. As can be seen, the positions at which $\Omega$ peaks in the regions of

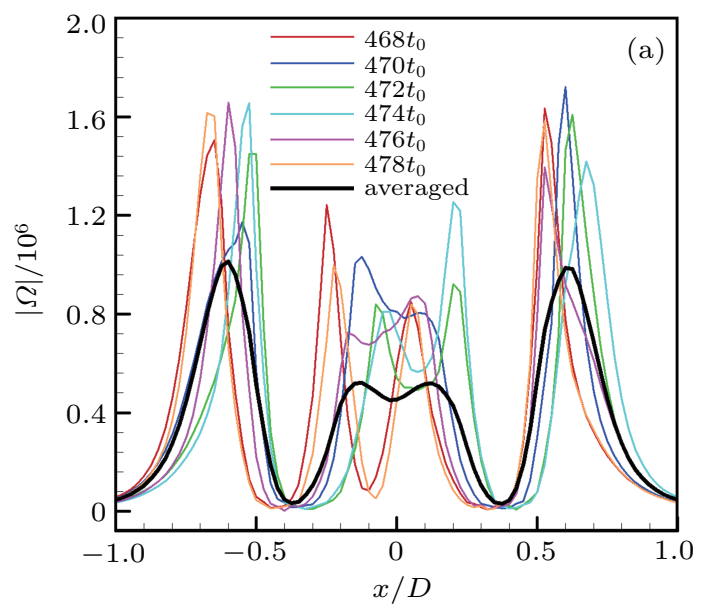

$-1.0<x / D<0.4$ and $0.4<x / D<1.0$ indicate the jet boundaries. The drifting of peak positions is thus corresponding to the oscillation of shock cells. Therefore, the positions with the maximum value of $\Omega$ in the radical direction at different time are carefully identified to quantitatively examine the shock oscillation phenomenon. Figure 13 shows the statistical results for NPR $=5.60$.

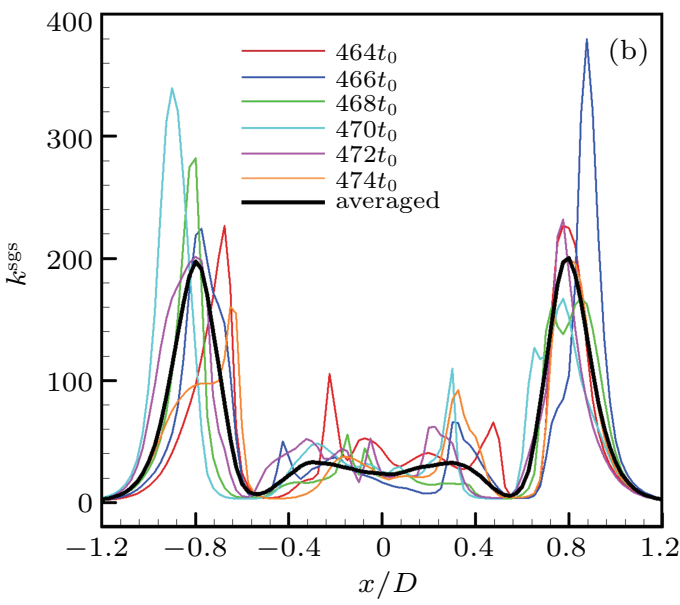

Fig. 12. (color online) Radial profiles of vorticity magnitude $\Omega$ sub-grid scale turbulent kinetic energy $k^{\text {sgs }}$ at (a) NPR $=5.60$ at $y / D=$ 6 , and (b) NPR $=9.34$ at $y / D=10$. The instantaneous curves in panel (a) are consistent with those in Figs. 7(b) -7 (g), successively.
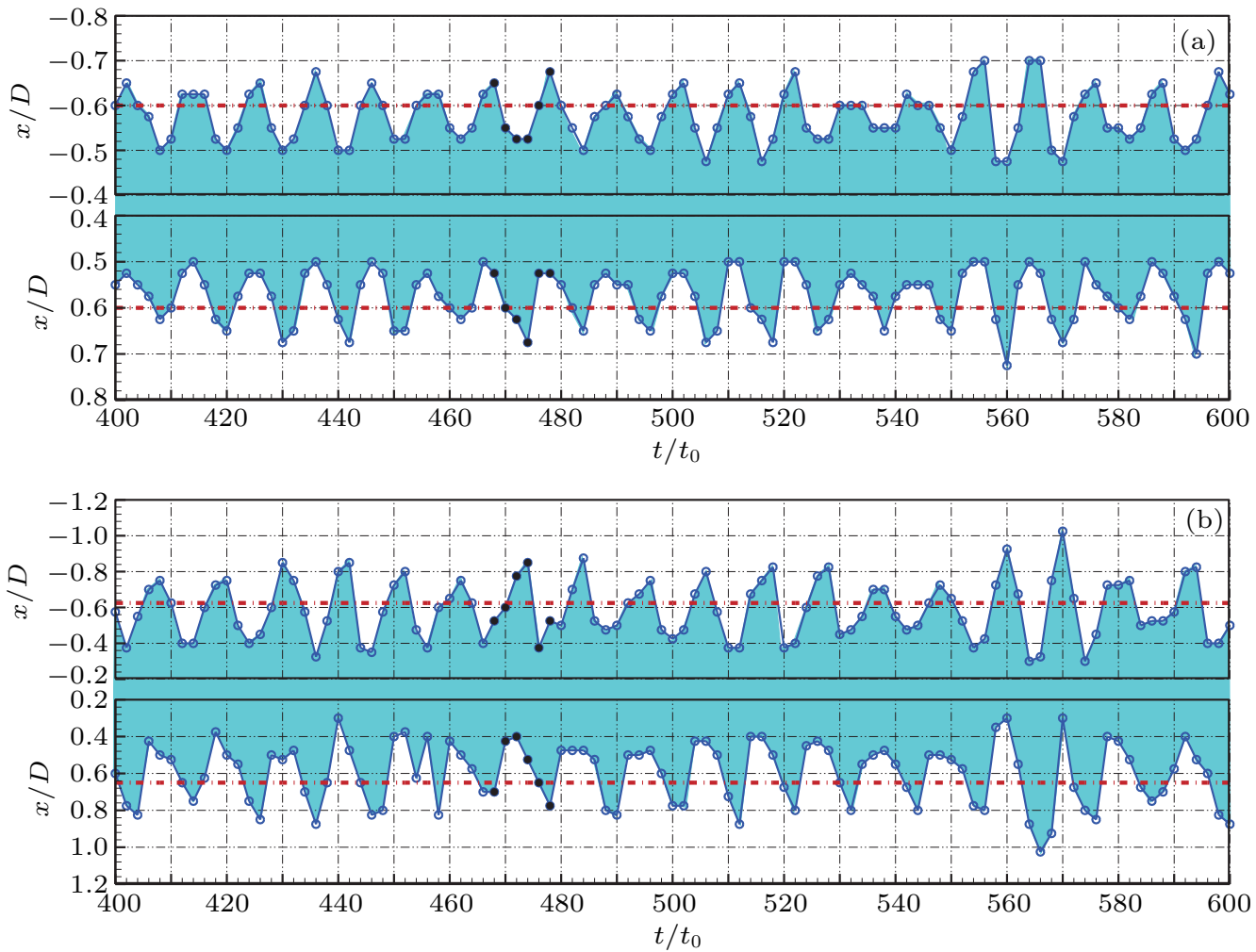

Fig. 13. (color online) Radial positions of maximum value of $\Omega$, revealing shock oscillation phenomenon for NPR $=5.60$ at $y / D$ of (a) 6 and (b) 8 . The empty circles with black solid lines correspond to those in Figs. 7(b)-7(g) as well as the instantaneous curves in Fig. 12(a), successively.

From Fig. 13, the shock oscillates almost sinusoidally around the time-averaged position, which is in accordance with the antisymmetric screech mode of the jet. Panda ${ }^{[15]}$ simply used the screech cycle to describe the shock motion versus time. André et al. ${ }^{[20]}$ demonstrated that the shocks oscillate at the screech frequency by comparing the spectra of the nearfield microphone signal with the spectra of the signal containing the shock locations. Figure 13 shows that most of shock oscillation cycles contain six points and the corresponding cycle is about $T_{1} \approx 10 t_{0}=25.0 \mu \mathrm{s}$, which is close to one wave 
period of the shock screech period $1 / f_{\mathrm{s}}=1 /\left(37.086 \times 10^{3}\right) \approx$ $26.96 \mu \mathrm{s}$. This demonstrates the direct evidence for the coincidence between the shock oscillation cycle and the screech frequency. In addition, a close examination shows that there are some shock oscillation cycles that contain only five points, implying a shorter cycle period of about $T_{1} \approx 8 t_{0}=20.0 \mu \mathrm{s}$, which is close to one wave period of the secondary shock screech $1 / f_{2 s}=1 /\left(45.695 \times 10^{3}\right) \approx 21.88 \mu$ s. This indicates that the shock cells inside the jet alternately oscillate at two different screech frequencies of $f_{\mathrm{s}}$ and $f_{2 \mathrm{~s}}$. The small differences between the oscillation cycle period and the wave period of the screech tone may be caused by insufficient time sampling rate (the instantaneous LES results are saved every $\left.2 t_{0}=5.0 \mu \mathrm{s}\right)$. The external shock oscillation reflects the internal helical motion of the jet core. From the present finding, it can be inferred that both the peak frequencies of $f_{\mathrm{s}}$ and $f_{2 \mathrm{~s}}$ are the valid screech frequencies that correspond to different helical modes, and the jet switches the motion modes between the two helical modes randomly for NPR $=5.60$ and 7.47 jets, as the conclusion ${ }^{[35]}$ obtained previously.
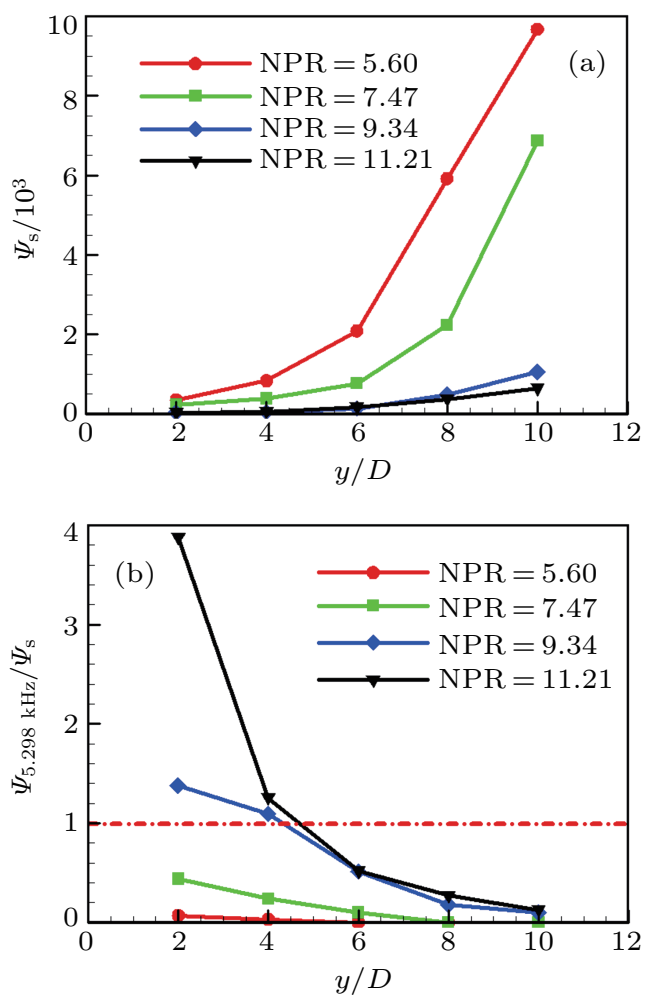

Fig. 14. (color online) (a) Amplitude of screech frequencies, and (b) amplitude ratio between the axisymmetric mode of $f=5.298 \mathrm{kHz}$ and screech tone. The screech frequencies presented here are $37.086 \mathrm{kHz}$, $34.437 \mathrm{kHz}, 31.787 \mathrm{kHz}$, and $29.801 \mathrm{kHz}$ for $\mathrm{NPR}=5.60,7.47,9.34$, and 11.21 ,respectively.

Figure 13 also shows that the shock oscillation amplitude is about $0.1 \mathrm{D}$ at $y / D=6$, and grows to around $0.2 \mathrm{D}$ at $y / D=8$. This quantitatively confirms the previous observation based on Fig. 7 that the oscillation increases for the shocks formed in the further down stream from the nozzle exit. It also agrees reasonably with the measurement by Panda. ${ }^{[15]}$ Figure 14(a) shows the amplitude of screech frequency, $\Psi_{\mathrm{s}}$, which increases with the distance of streamwise position increasing, as the shock oscillation magnitude. Meanwhile, the shock oscillation amplitude $\sim 0.2 D$ at $y / D=8$ for $\mathrm{NPR}=5.60$ is larger than $\sim 0.08 D$ for NPR $=9.34$ (as shown in Fig. 15), which is consistent with the screech amplitude trend, i.e., the screech amplitude $\Psi_{\mathrm{s}}$ is larger for the lower NPR. These observations confirm the conclusions by André et al. ${ }^{[20]}$ based on the schlieren photographs, i.e., the shock oscillation magnitude is strongly related to the screech amplitude, probably being an increasing function of the screech amplitude.

Moreover, it is interesting to find from Fig. 13 that the shocks tend to oscillate more intensively toward the jet centerline. First, the shock oscillation amplitude on the centerline side appears to be larger than that away from the jet centerline, no matter whether the value of $y / D$ is 6 or 8 , and no matter whether the half shaft is the positive half shaft $(x / D>0)$ or negative half shaft $(x / D<0)$. Second, there are generally more time sampling points (usually $3-4$ ) on the centerline side during one oscillation cycle. This finding is in accordance with the observation by Panda, ${ }^{[15]}$ that is, the shocks inside the underexpanded jets move most in the jet core and least in the shear layer spatially.

Besides the vorticity magnitude $\Omega$, the sub-grid scale turbulent kinetic energy $k^{\text {sgs }}$ also peaks at the jet boundary, and can be used to track the shock locations as well. Figure 12(b) shows the instantaneous radial profiles of $k^{\text {sgs }}$ at different time, and figure 15 presents the jet boundaries identified based on $k^{\text {sgs }}$ for NPR $=9.34$. The overall characteristics of shock oscillation for NPR $=9.34$ are similar to those for $\mathrm{NPR}=5.60$. For example, the shock oscillation amplitude is larger for higher streamwise position. It is also found that the shocks appear to oscillate more intensively toward the jet centerline. However, the periodical characteristic of shock oscillation for $\mathrm{NPR}=9.34$ is more complex than that for $\mathrm{NPR}=5.60$. There seems to be more concentrated time sampling points near the time-averaged shock positions. The shock also appears to oscillate more quickly for $\mathrm{NPR}=9.34$ than for $\mathrm{NPR}=5.60$. These may have two origins. First, the axisymmetric mode plays a more important role and takes effect within a much longer distance at a higher NPR. Figure 14(b) presents the amplitude ratios between axisymmetric mode and screech tone, $\Psi_{5.298 \mathrm{kHz}} / \Psi_{\mathrm{S}}$, for different NPRs. As can be seen, the amplitude ratio $\Psi_{5.298 \mathrm{kHz}} / \Psi_{\mathrm{s}}$ is generally large in the near-nozzle region and decreases with being away from the streamwise position. This agrees reasonably with the findings by Mattingly ${ }^{[50]}$ on low Reynolds jets based on the inviscid stability theory, i.e., the dominant disturbance in the very near field of jets is an axisymmetric one (azimuthal wavenumber $m=0$ ), while the single helical disturbance $(\mid m=1)$ is dominant in the further 
down stream. Meanwhile, the amplitude ratio $\Psi_{5.298 \mathrm{kHz}} / \Psi_{\mathrm{s}}$ is larger for higher NPR at different streamwise positions. These observations suggest that the axisymmetric mode affects the oscillation of shock cells in radial direction except its domination in Mach disk oscillation in the streamwise direction, especially for higher NPR. As a result, the shock oscillation for higher NPR looks more symmetric, which implies that more time sampling points are located near the time-averaged shock positions. Second, an increase of the effect of helical modes with higher frequency and larger wave numbers, such as the double helical modes $(|m|=2)$, on the jet flow for higher NPR, may result in a faster switching in shock oscillation cycle.
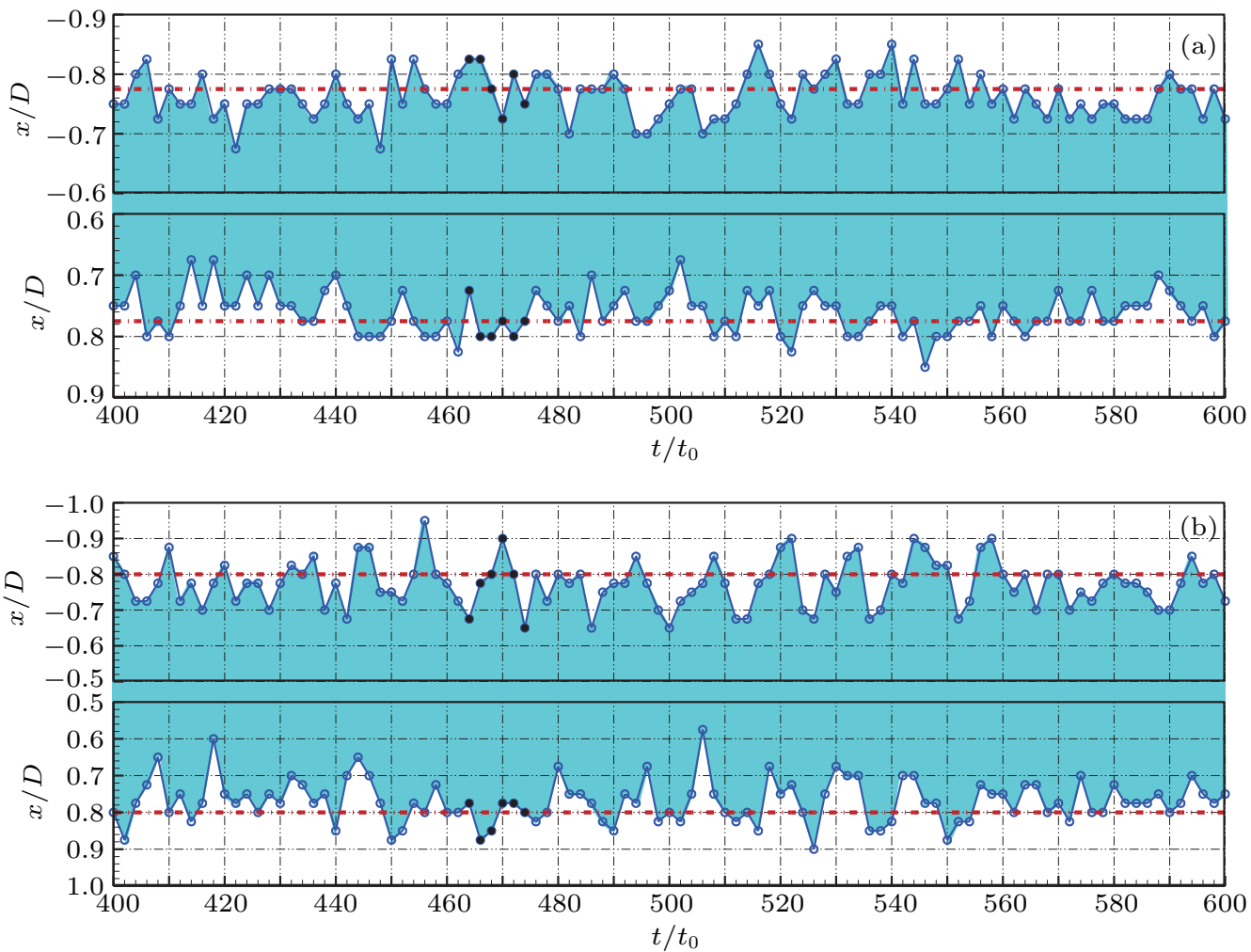

Fig. 15. (color online) . Radial positions of maximum value of $k^{\text {sgs }}$, revealing the shock oscillation phenomenon for NPR $=9.34$ at $y / D$ of (a) 8 and (b) 10 . The empty circles with black solid lines correspond to the instantaneous curves in Fig. 12(b).

\subsection{Effect of mesh resolution and subgrid-scale model}

In general, the fine mesh is able to capture more smallscale turbulence structures, but its computational cost is higher. Likewise, the one-equation SGS model has less advantage in computational efficiency because more equations are solved than the zero equation models, such as the Smagorinsky model. Therefore, the Smagorinsky model ${ }^{[51,52]}$ is also used for simulating the NPR $=5.60$ jet, and the effect of grid resolution and SGS model on revealing the unsteady shock motion are investigated in this section.
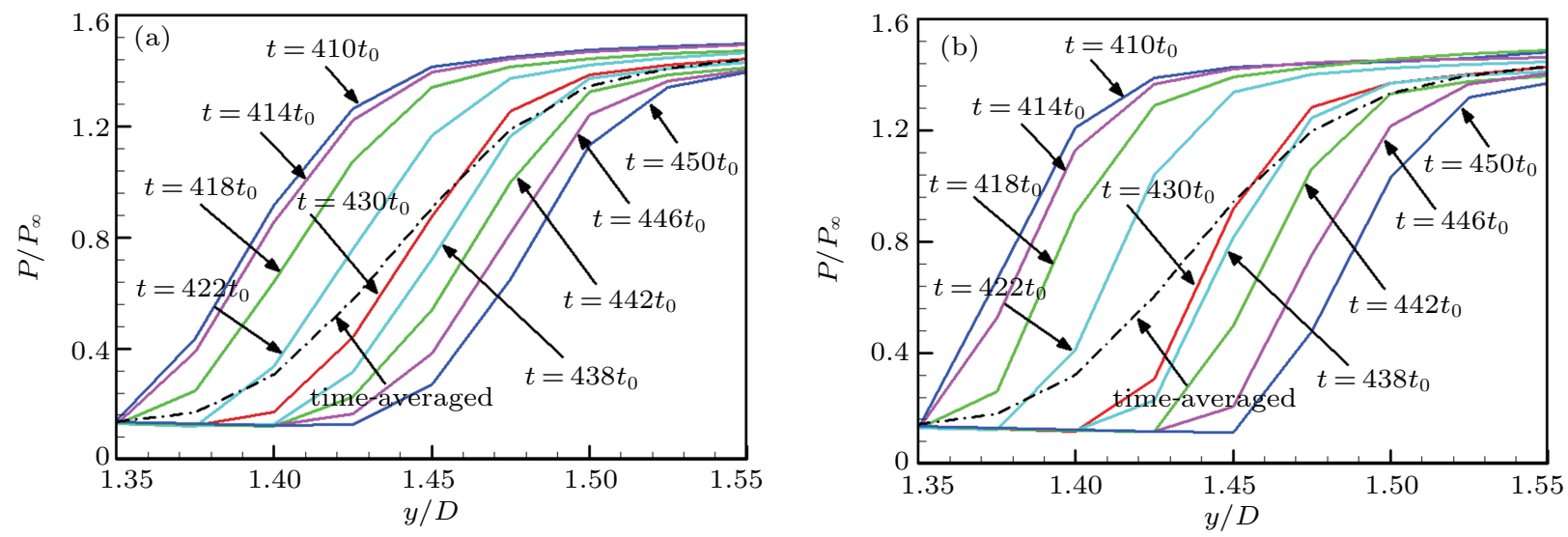

Fig. 16. (color online) Instantaneous pressures along the jet centerline obtained under different simulation conditions for NPR $=5.60$, indicating the Mach disk motion in half the oscillation cycle, by using (a) one equation SGS model with coarse mesh and (b) Smagorinsky model with fine mesh. 
Figures 16(a) and 16(b) show the Mach disk oscillations for NPR $=5.60$ jet, revealed using the coarse mesh and the Smagorinsky model, respectively, where the time is the same as that presented in Fig. 9(a). Meanwhile, like Fig. 11(a), the cross spectrum and relative phase of pressure fluctuation at $y / D=2$ obtained by the coarse mesh and the Smagorinsky model are plotted in Fig. 17. As can be seen, the oscillation characteristics of the Mach disk are also well captured by the coarse mesh and the Smagorinsky model, i.e., the Mach disk oscillates at the same cycle around $76 t_{0}=190 \mu$ s and moves more quickly in the middle region. Moreover, the axisymmetric mode of $5.298 \mathrm{kHz}$ that dominates the Mach disk oscillation also exists in the spectra obtained by the coarse mesh and Smagorinsky model, which may be related to the nozzle geometry used in the present LES as discussed previously. However, the oscillation amplitude of Mach disk presented in Fig. 16 seems to be smaller than that shown in Fig. 9(a), especially for the results obtained using the coarse mesh.
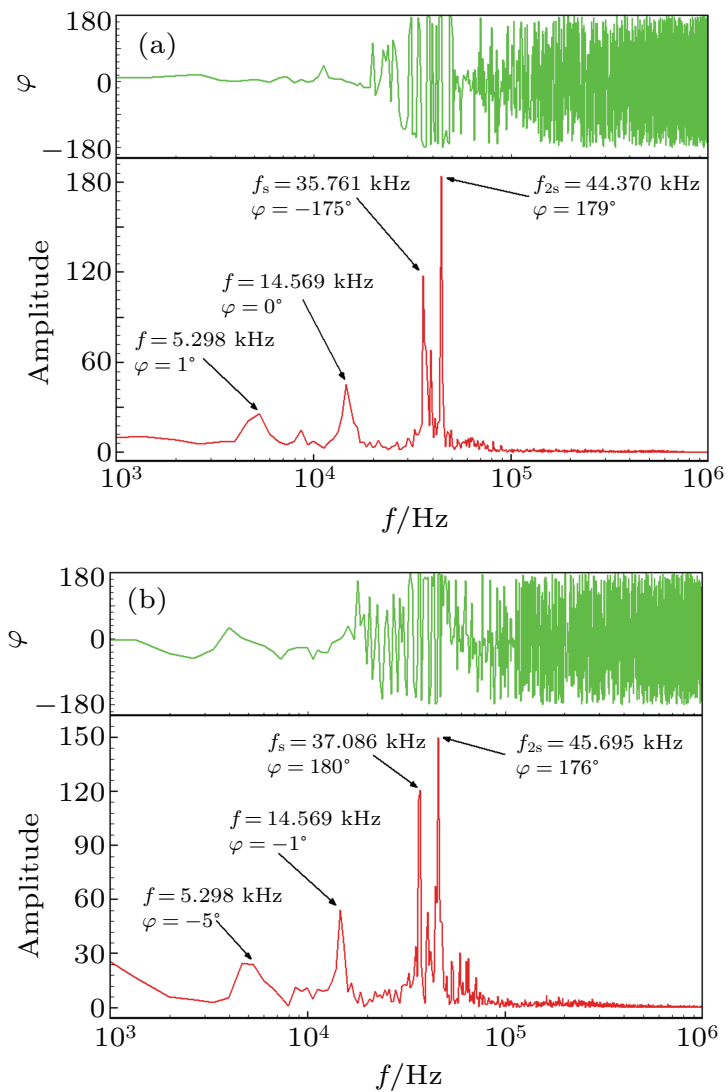

Fig. 17. (color online) Cross spectra and relative phases of pressure fluctuation on either side of the jets obtained under different simulation conditions for NPR $=5.60$ by using (a) one equation SGS model with coarse mesh and (b) Smagorinsky model with fine mesh.

As also shown in Fig. 17, the two helical modes that dominate the shock oscillation in the radial direction are captured by the coarse mesh, and take values of $35.761 \mathrm{kHz}$ and $44.370 \mathrm{kHz}$, respectively, which are slightly smaller than $37.086 \mathrm{kHz}$ and $45.695 \mathrm{kHz}$ resolved by the fine mesh. Meanwhile, the Smagorinsky model captures the two helical modes as well, and the peak frequencies are the same as those obtained by the one-equation model, i.e., $37.086 \mathrm{kHz}$ and $45.695 \mathrm{kHz}$.
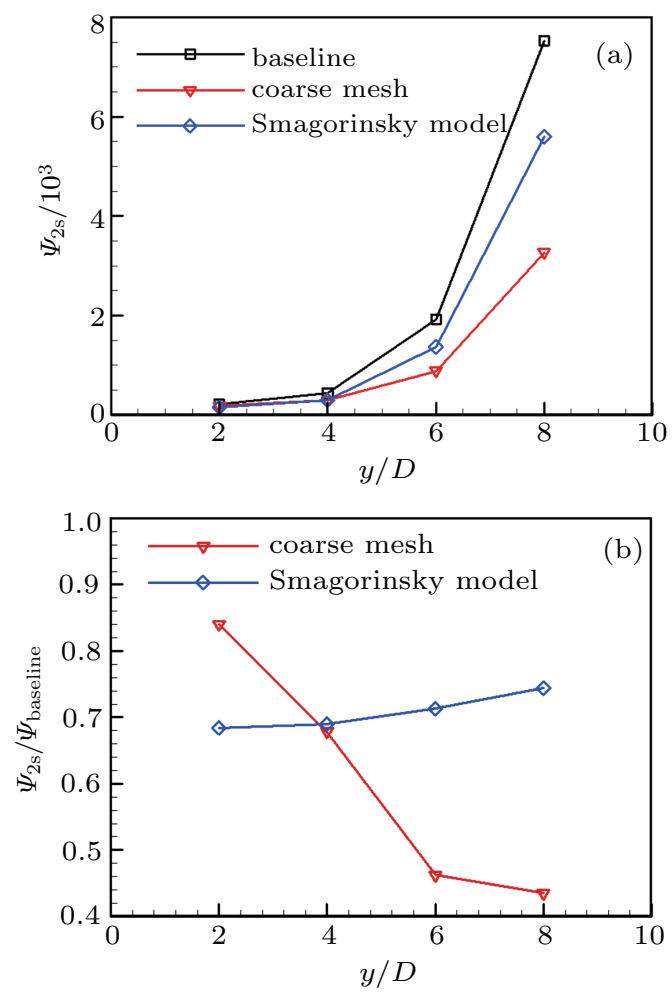

Fig. 18. (color online) Comparison of shock screech amplitude obtained under different simulation conditions for NPR $=5.60$ : (a) amplitude of shock screech frequency $f_{2 \mathrm{~s}}$, (b) non-dimensional amplitude ratio of shock screech tone $f_{2 \mathrm{~s}}$.

In the previous section, it is indicated that the magnitude of shock oscillation in the radial direction is an increasing function of the screech amplitude. Therefore, the amplitudes of shock screech frequency $f_{2 \mathrm{~s}}$ signals captured using different simulation conditions are compared in Fig. 18(a) to characterize the radial shock oscillation magnitude. As can be seen, the overall profiles of the shock screech amplitudes obtained based on the coarse mesh and Smagorinsky model are similar to those in the baseline case (the simulation with the fine mesh and one-equation model) as well as the results presented in Fig. 14 (a), i.e., the screech amplitude $\Psi_{2 \mathrm{~s}}$ increases with the distance of streamwise position decreasing. However, the simulations using coarse mesh and Smagorinsky model produce relatively small $\Psi_{2 \mathrm{~s}}$ values. In particular, the $\Psi_{2 \mathrm{~s}}$ values for the coarse mesh take the smallest values at $y / D=6$ and 8. This observation suggests that the coarse mesh tends to predict a much weaker radial shock oscillation, just as predicting a weaker Mach disk oscillation shown in Fig. 16. In order to further check how far it deviates from the screech amplitude obtained based on the one-equation model with fine mesh, the screech amplitude of the baseline case is chosen as the reference value, and the non-dimensional amplitude ratio $\Psi_{2 \mathrm{~s}} / \Psi_{\text {baseline }}$ using the Smagorinsky model with coarse mesh is 
computed numerically, and the results are plotted in Fig. 18(b). It is clear that the amplitude ratio for the coarse mesh decreases with the distance of streamwise position increasing. This trend may be explained by the increase of dissipation due to the decay of grid resolution along the streamwise direction. For the simulation using the Smagorinsky model, the amplitude ratio is about 0.68 at $y / D=2$, and increases gradually to 0.74 at $y / D=8$. These findings indicate that the disadvantage of Smagorinsky model in revealing the unsteady shock motions in underexpanded jets is not so remarkable, because of the considerable performances in capturing the frequency and magnitude of shock oscillation. On the other hand, it appears that the shock oscillation characteristics resolved by the LES, including the oscillation frequency and magnitude, rely more on the grid resolution used.

\section{Conclusions}

In this work, the oscillatory characteristics of shocks formed inside highly underexpanded jets $(\mathrm{NPR}=5.60,7.47$, 9.34, and 11.21) issuing from a convergent nozzle are studied based on high-resolution LES data. Two types of shock oscillations are observed. One is the Mach disk oscillation in the streamwise direction, and the other is the shock oscillation in the radial direction that has been previously measured using schlieren photograph. The instantaneous contours of density gradient are used to qualitatively visualize the shock motion. The pressure profiles along the jet centerline are examined to quantitatively characterize the Mach disk oscillation, while the shock motions in the radial direction are addressed based on the jet boundary tracking through the radial profilies of vorticity and SGS TKE. The possible reasons for shock oscillations are discussed. In addition, the effect of grid resolution and SGS turbulence models used in the LES to reveal the shock oscillation characteristics are examined. The main conclusions are summarized as follows.

1) The Mach disk shows an up and down motion in the streamwise direction, and moves quickly in the middle of the oscillation region but slowly at the top and bottom end.

2) The oscillation cycles of Mach disk are the same for different NPRs, and are dominated by the axisymmetric mode of $5.298 \mathrm{kHz}$ under the current nozzle geometry designed.

3) The shock cells undergo considerable distortion and show some oscillation in the radial direction. The oscillation is more intense toward the jet centerline and less intense in the jet shear layer.

4) The shock oscillation amplitude in the radial direction increases along the streamwise direction, and is larger for lower NPR, implying that it is an increasing function of screech amplitude.
5) The shock oscillation cycle in the radial direction switches randomly between the two screech frequencies of $f_{\mathrm{s}}$ and $f_{2 \mathrm{~s}}$ for NPR $=5.60$ and 7.47 .

6) At higher NPR values (9.34 and 11.21), the general characteristics of shock motion in the radial direction are similar to thoase at the low NPR values, but the oscillation periodicity is more complex because the effect of the axisymmetric mode and helical mode strengthen with wave number increasing.

In addition, the shock oscillation characteristics are also captured by the coarse mesh and the Smagorinsky model. The peak frequencies obtained by the Smagorinsky model are the same as those by the one-equation model, and the amplitude ratio of screech tones increases with the distance of streamwise position increasing. However, the oscillation frequency captured by the coarse mesh is slightly smaller than that by the fine mesh, and the coarse mesh also tends to predict a much weaker shock oscillation magnitude.

\section{References}

[1] Chen Y X, Chen K, You Y X and Hu T Q 2013 Acta Phys. Sin. 62 114701 (in Chinese)

[2] Xu M Y, Tong X Q, Yue D T, Zhang J P, Mi J C, Nathan G J and Kalt P A M 2014 Chin. Phys. B 23124703

[3] Zhang J P, Xu M Y and Mi J C 2014 Chin. Phys. B 23044704

[4] Li Y M, Li B K, Qi F S and Wang X C 2017 Chin. Phys. B 26024701

[5] Yan H, Wang F J, Li C C and Huang J 2017 Chin. Phys. B 26044701

[6] Tam C K W 1995 Annu. Rev. Fluid Mech. 2717

[7] Raman G 1998 Prog. Aerosp. Sci. 3445

[8] Franquet E, Perrier V, Gibout S and Bruel P 2015 Prog. Aerosp. Sci. 77 25

[9] Prandtl L 1904 Physikalische Zeitschrift 4599

[10] Pack D C 1950 Quarterly J. Mech. Appl. Math. 3173

[11] Powell A 1953 Proc. Phys. Soc. B 661039

[12] Sherman P M, Glass D R and Duleep K G 1976 Appl. Sci. Res. 32283

[13] Tam C K W and Tanna H K 1982 J. Sound Vib. 81337

[14] Tam C K W 1990 J. Sound Vib. 14055

[15] Panda J 1998 J. Fluid Mech. 363173

[16] Panda J 1999 J. Fluid Mech. 37871

[17] Panda J and Seasholtz R G 1999 Phys. Fluids 113761

[18] Raman G 1997 J. Fluid Mech. 330141

[19] Panda J, Raman G and Zaman K B M Q 1997 Third AeroAcoust. Conf., May 12-14, 1997, Georgia, USA, AIAA 1997

[20] André B, Castelain T and Bailly C 2011 AIAA J. 491563

[21] André B, Castelain T and Bailly C 2012 AIAA J. 502017

[22] André B, Castelain T and Bailly C 2014 Shock Waves 2421

[23] Edgington-Mitchell D, Honnery D R and Soria J 2014 Phys. Fluids 26 096101

[24] Rogers T, Petersen P, Koopmans L, Lappas P and Boretti A 2015 Int. J. Hydrogen Energy 401584

[25] Singh A and Chatterjee A 2007 Shock Waves 17263

[26] Berl, J, Bogey C and Bailly C 2007 Phys. Fluids 19075105

[27] Vuorinen V, Yu J, Tirunagari S, Kaario O, Larmi M, Duwig C and Boersma B J 2013 Phys. Fluids 25016101

[28] Vuorinen V, Wehrfritz A, Duwig C and Boersma B J 2014 Fuel 130 241

[29] Hamzehloo A and Aleiferis P G 2014 Int. J. Hydrogen Energy 3921275

[30] Hamzehloo A and Aleiferis P G 2016 Int. J. Hydrogen Energy 416544

[31] Li X P, Wu K, Yao W and Fan X J 2016 Int. J. Hydrogen Energy 41 5151

[32] Donaldson C D and Snedeker R S 1971 J. Fluid Mech. 45281 
[33] Belan M, De P S and Tordella D 2010 Phys. Rev. E 82530

[34] Dubs P, Khalij M, Benelmir R and Tazibt A 2011 Mech. Res. Commun. 38267

[35] Li X P, Yao W and Fan X J 2016 AIAA J. 543191

[36] Chakravarthy V and Menon S 2001 Combustion Sci. Technol. 162175

[37] Chase M W 1974 J. Phys. Chem. Ref. Data 3311

[38] Greenshields J G, Weller H G, Gasparini L and Reese J M 2010 Int. J. For Numer. Methods Fluids 63

[39] Kurganov A and Tadmor E 2000 J. Comput. Phys. 160241

[40] Jasak H, Weller H and Gosman A 1999 Int. J. Numer. Methods Fluids 31431

[41] Baba-Ahmadi M H and Tabor G 2009 Comput. Fluids 381299

[42] Liu J, Ramamurti R, Munday D, Gutmark E, Kailasanath K and Lohner R 2009 AIAA J. 471849
[43] Gorle C, Gamba M and Ham F 2010 Center For Turbulence Res. Annu. Res. Briefs, 2010, Center for Turbulence Research, Stanford, CA

[44] Dauptain A, Cuenot B and Gicquel Y M 2010 AIAA J. 482325

[45] Kawai S and Lele K 2010 AIAA J. 482063

[46] Rana Z A, Thornber B and Drikakis D 2011 Phys. Fluids 23046103

[47] Hamzehloo A and Aleiferis P G 2016 Int. J. Heat Fluid Flow 61711

[48] Gribben B, Badcock K and Richards B 2000 AIAA J. 38275

[49] Mate B, Graur I A, Elizarova T, Chirokov I, Tejeda G, Fernández J M and Montero S 2001 J. Fluid Mech. 426177

[50] Mattingly G E 1974 J. Fluid Mech. 65541

[51] Smagorinsky J 1963 Mon. Weather Rev. 9199

[52] Erlebacher G, Hussaini M Y, Speziale C G and Zang T A 1992 J. Fluid Mech. 238155 